\title{
Bandwidth Efficient Channel Estimation using Super-imposed Pilots for OFDM systems
}

\author{
Kaushik Josiam, Dinesh Rajan \\ Department of Electrical Engineering \\ Southern Methodist University \\ Dallas, TX 75275, USA \\ Email: \{kjosiam, rajand $\} @$ engr.smu.edu
}

\begin{abstract}
This paper explores channel estimation using superimposed pilots for OFDM systems. The pilots are added linearly to the modulated symbol at a fraction of the total transmit power, making the method spectrally efficient. We present a series of receivers in which complexity is traded for performance; in particular, a receiver that achieves the theoretically lowest BER but has high complexity and a low complexity receiver that shows near optimal performance. We show by simulations that the performance of the receiver is independent of the channel's Doppler frequency and delay spread. We also analytically compute a bound for the bit error rate performance of the proposed receiver.
\end{abstract}

Index Terms-OFDM, super-imposed pilots, Channel Estimation, Viterbi.

\section{INTRODUCTION}

Orthogonal Frequency Division Multiplexing (OFDM) [1] is a multi-carrier modulation technique that comes with an inherent promise of spectrally efficient high data rate transmission and multi-path effect mitigation in wideband wireless systems. The available bandwidth is split between the carriers and the bandwidth allocated to each sub-carrier is narrow enough for the channel to be considered flat fading over it. Accordingly, it's use has been standardized in high speed wireless - local area network (W-LAN) systems (for example, IEEE 802.11, HIPERLAN2) and for digital audio and video broadcasting (DAB and DVB) in Europe.

The block diagram detailing the data processing in an OFDM system is shown in Fig. 1. Information bearing symbols are modulated using a fixed constellations like QPSK or 16-QAM. The modulated symbols are then segmented into blocks of length $N$. Each block of symbols is transformed using inverse discrete Fourier transform (IDFT) to generate a time domain symbol block. The last $N_{c p}$ symbols in the block containing time-domain symbols are repeated at its beginning forming the cyclic prefix $(\mathrm{CP})$. The symbols are then sequentially transmitted over a frequency selective channel. If the length of the channel memory is less than the cyclic prefix, the distortion due to convolution of the transmitted symbols with the channel is limited to the symbols in the CP and inter-symbol interference (ISI) is avoided. At the receiver, the $\mathrm{CP}$ is removed and the symbol is transformed back into the frequency domain using discrete Fourier transform (DFT) filtering. The transmitted symbols are then recovered from the

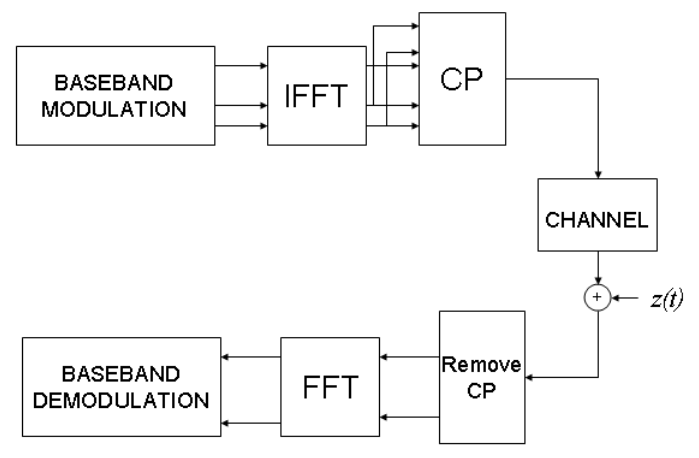

Fig. 1. OFDM Block Diagram.

frequency domain symbols using a combination of channel estimation and symbol detection.

Although ISI has been avoided using CP, the data on each subcarrier is weighted differently due to the frequency selectivity of the channel. For coherent symbol detection, knowledge of each subchannel's gain and phase is critical. Often in practice, training symbols known as pilots that are known to the receiver a priori, are multiplexed on to the data stream for channel estimation [2]. For example, in 802.11 networks, a few specific sub-carriers are dedicated for transmitting pilot symbols. However, the addition of pilots cause bandwidth expansion and hence reduces data rate. For spectrally efficient transmission, blind channel estimation methods that do not use pilot symbols have been proposed. Existing blind methods primarily use the statistics of the received signal to estimate the channel [3]-[5]. Though spectrally advantageous, blind methods converge slower and involve high complexity.

This paper focuses on a spectrally efficient pilot arrangement for channel estimation in OFDM systems. The pilots are linearly added on the data and hence called super-imposed pilots. The concept of super-imposed pilots for simultaneous data transmission and channel estimation was first proposed for analog communication systems in a 1965 paper [6]. Farhang-Bouroujeny in 1995 [7] applied super-imposed pilots for digital communication systems. There is a considerable body of work on super-imposed pilots for communication systems [8]-[11]. Most of the proposed algorithms are derived 
based on an underlying channel model and are hence limited in the range of channel conditions over which they can be used.

In this paper, we propose a joint channel estimation and symbol detection algorithm based on maximum likelihood sequence estimation (MLSE) principles. A similar approach was used for single carrier systems in [8], where they used prediction to update the channel estimate at each time instant. Here, we take advantage of the OFDM structure and the blockfading channel to derive the optimal channel estimate that minimizes the mean-squared error metric. The proposed receiver's performance is independent of the channel parameters, namely the coherence time and the root mean square (RMS) delay spread, as long as there is no ISI. Hence the algorithm can be applied to both slow and fast fading channels. We also derive an analytical bound for the bit error rate (BER) of the proposed receiver with QPSK modulation and present results for a low complexity near optimal receiver.

The rest of the paper is organized as follows. The system description along with the channel model used is given in Section II. The channel estimation algorithm along with the receiver design is described in Section III. The numerical results are given in Section IV followed by conclusions in Section V.

\section{System Description}

\section{A. Transmission Strategy}

At the transmitter, a known pseudo-noise (PN) sequence is added to the unknown data symbols as pilots. Let $u_{n, t} \in \mathbf{C}$ and $p_{n, t} \in \mathbf{C}$ represent the data and the pilot symbols respectively, where $n$ is the subcarrier index and $t$ is the time index. The data sequence belong to a $M$-ary alphabet depending on the modulation chosen. The assumed statistical properties of data and pilot symbols are as follows:

- The data and pilot sequences are each i.i.d and zero mean i.e., $E\left[u_{n, t}\right]=E\left[p_{n, t}\right]=0$

- The data and pilot sequences are uncorrelated i.e., $E\left[u_{n, t} p_{n, t}^{*}\right]=0$.

Let $\rho$ denote the fraction of the transmit energy $E_{s}$ allocated to the pilot symbols. Then, the average energy of the pilot symbols is $E_{p}=E\left[\left|p_{n, t}\right|^{2}\right]=\rho \cdot E_{s}$ and the average data energy is $E_{u}=E\left[\left|u_{n, t}\right|^{2}\right]=(1-\rho) \cdot E_{s}$. The pilot symbols are super-imposed on the data at a fraction of the total transmit power $\rho$ at all points in the time-frequency plane and the arrangement is shown in Fig 2.

\section{B. Received Signal}

The received signal $r_{n, t}$, after the FFT block in Fig. 1, at the $n^{\text {th }}$ subcarrier and time instant $t$, is given by

$$
r_{n, t}=\left(u_{n, t}+p_{n, t}\right) f_{n}+z_{n, t}
$$

where, $f_{n} \in \mathbf{C}$ is a multiplicative fading coefficient of the channel and $z_{n, t} \in \mathbf{C}$ is the zero mean AWGN with power $\sigma_{z}^{2}$.

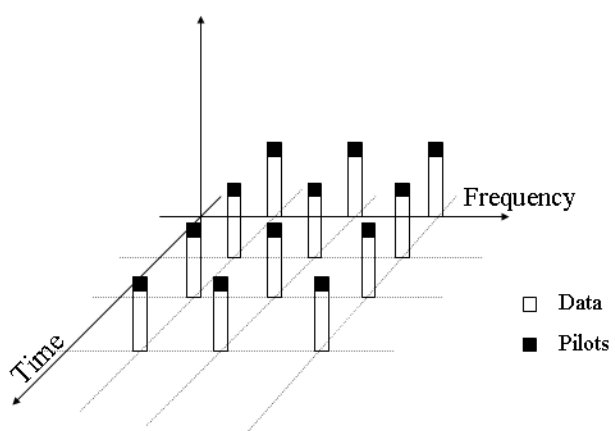

Fig. 2. Pilots super-imposed over data at all temporal and spectral locations.

\section{Channel Model}

The multiplicative fading coefficient, $f_{n}$, is the frequency response of the channel evaluated at the $n^{\text {th }}$ sub-carrier. The channel is modeled in the time-domain as a linear timevarying (LTV) filter with varying lengths that depend on the RMS delay spread of the channel. The coefficients of the LTV filter are assumed to be complex Gaussian with zero mean and variances $\sigma_{k}^{2}$ which decay exponentially and is given by,

$$
\sigma_{k}^{2}=\sigma_{0}^{2}\left(e^{-\frac{k}{F_{s} \tau_{d}}}\right) \quad k=0, \ldots, K
$$

where, $\sigma_{0}^{2}=1-e^{\frac{-1}{F_{s} \tau_{d}}}, F_{s}$ is the channel bandwidth, $\tau_{d}$ is the RMS delay spread of the channel and $K+1$ is the number of taps in the channel filter. Typically $K$ is chosen as $\left\lceil 10 F_{s} \tau_{d}\right\rceil[12]$. The channel thus generated remains fixed for the duration of $T_{c}$ symbols, and changes in filter coefficients after $T_{c}$ symbols are independent, i.e., a standard block fading channel. It should be noted that this channel model is used only to generate the received signal; the channel model is not used in deriving the channel estimation and data detection algorithm.

\section{OPTIMAL RECEIVER FOR BLOCK FADING CHANNELS}

The received signal for the $n^{t h}$ subcarrier over each subinterval of length $T_{c}$ symbols can be equivalently written in vector form as,

$$
\mathbf{r}_{n}=\left(\mathbf{u}_{n}+\mathbf{p}_{n}\right) f_{n}+\mathbf{z}_{n}
$$

where $\mathbf{r}_{n}, \mathbf{u}_{n}, \mathbf{p}_{n}$ and $\mathbf{z}_{n}$ are $T_{c}$-dimensional vectors representing the received data, transmitted data, pilot symbols and AWGN symbols respectively.

Since the noise vector $\mathbf{z}_{n}$ is complex Gaussian with zero mean and variance $\sigma_{z}^{2}$, the likelihood function that a given data hypotheses $\tilde{u}_{n, T_{c}}, \tilde{u}_{n, T_{c}-1}, \ldots, \tilde{u}_{n, 1}$ was transmitted on the $n^{\text {th }}$ subcarrier and that the channel estimate is $\tilde{f}_{n}$, can be written as,

$$
g_{z}\left(\mathbf{r}_{n} \mid \tilde{\mathbf{u}}_{n}, \tilde{f}_{n}\right)=\left(\frac{1}{\sqrt{2 \pi \sigma_{z}^{2}}}\right)^{T_{c}} \prod_{i=1}^{T_{c}} e^{-\frac{\left|r_{n, i}-\left(\tilde{u}_{n, i}+p_{n, i}\right) \tilde{f}_{n}\right|^{2}}{2 \sigma_{z}^{2}}}
$$


Maximizing the likelihood function is equivalent to minimizing the exponent term in (4). A simple approach to find the ML sequence is to exhaustively and recursively search among all $M^{T_{c}}$ possible hypotheses to find the one that minimizes the mean squared error (MSE) metric $\Lambda$ for each subcarrier $n$. This MSE is given by,

$$
\begin{array}{r}
\Lambda\left(\tilde{u}_{n, t-T_{c}}, \tilde{u}_{n, t-T_{c}+1}, \ldots, \tilde{u}_{n, 1}\right)= \\
\frac{1}{T_{c}} \sum_{i=1}^{T_{c}}\left|r_{n, i}-\left(\tilde{u}_{n, i}+p_{n, i}\right) \cdot \tilde{f}_{n}\right|^{2}
\end{array}
$$

Similarly, for a given data hypotheses $\left\{\tilde{u}_{n, T_{c}}, \tilde{u}_{n, T_{c}-1}, \ldots, \tilde{u}_{n, 1}\right\}$, the optimum channel hypothesis $\tilde{f}_{n}$ that minimizes the minimum mean squared error can be shown to be,

$\tilde{f}_{n}\left(\tilde{u}_{n, t-T_{c}}, \tilde{u}_{n, t-T_{c}+1}, \ldots, \tilde{u}_{n, 1}\right)=\frac{1}{T_{c}} \sum_{i=1}^{T_{c}} \frac{r_{n, i}\left(\tilde{u}_{n, i}+p_{n, i}\right)^{*}}{\left|\tilde{u}_{n, i}+p_{n, i}\right|^{2}}$

The computational complexity of the algorithm (i.e., the number of arithmetic operations needed for channel estimation and symbol detection) is $O\left(N M^{T_{c}}\right)$. It can be seen that for slow-varying channels where $T_{c}$ is very high, the complexity increases exponentially. Also, for higher order modulations that are typically used in high data rate environments, the complexity is high. Instead, we use a computationally efficient search algorithm like the Viterbi, to find the maximum likelihood sequence.

\section{Viterbi Receiver}

The received vector $\mathbf{r}_{n}$ is processed over a small sliding time window of length $L$ using the Viterbi algorithm [13] on a suitable trellis. For window length $L$, there are $M^{L}$ possible data hypotheses and hence $M^{L}$ states in the trellis as shown in Fig. 3. Each state has $M$ branches coming into and out of every state. The data hypotheses, $\left\{\tilde{u}_{n, t-L}, \ldots, \tilde{u}_{n, t-1}\right\}$ identify the state of the trellis. The channel hypothesis at any given time instant $t$ is now averaged over the $L$ data hypotheses associated with a particular state of the trellis and the data hypothesis $\tilde{u}_{n, t}$ of the current transition from that state. Since the sliding window is used to average the channel hypothesis over $L$ received symbols, it is also called as the averaging order. Accordingly, the channel hypothesis (6) is now replaced by,

$$
\begin{gathered}
\breve{f}_{n}\left(\tilde{u}_{n, t} / \tilde{u}_{n, t-L}, \ldots, \tilde{u}_{n, t-1}\right)= \\
\frac{1}{L+1} \sum_{l=0}^{L} \frac{r_{n, t-l}\left(\tilde{u}_{n, t-l}+p_{n, t-l}\right)^{*}}{\left|\tilde{u}_{n, t-l}+p_{n, t-l}\right|^{2}}
\end{gathered}
$$

The channel hypothesis $\breve{f}_{n}$ and the data hypotheses are used to calculate the MSE metric for the current transition at time instant $t$ as,

$$
\Lambda^{\prime}\left(\tilde{u}_{n, t} / \tilde{u}_{n, t-L}, \ldots, \tilde{u}_{n, t-1}\right)=\left|r_{n, t}-\left(\tilde{u}_{n, t}+p_{n, t}\right) \cdot \breve{f}_{n}\right|^{2}
$$

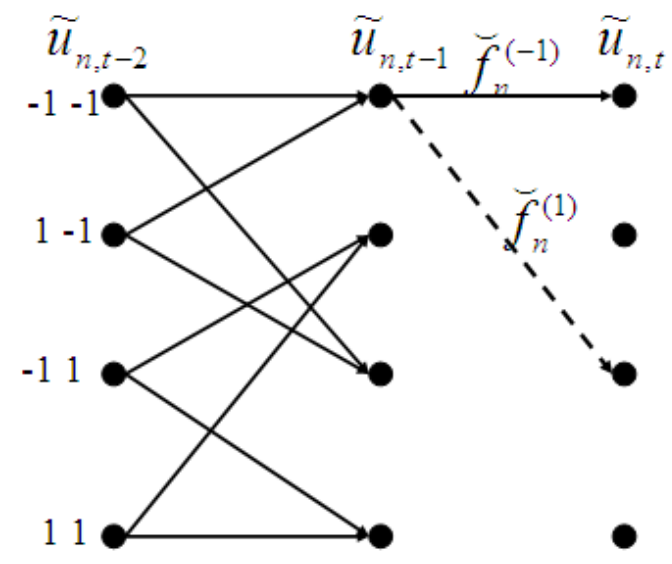

Fig. 3. Trellis showing the states and transitions for a modulation order $M=2$ and averaging order $L=2 . \breve{f}_{n}^{(-1)}$ and $\breve{f}_{n}^{(1)}$ are the computed channel hypotheses (7) for transitions corresponding to data hypothesis -1 and +1 respectively.

Since the noise is Gaussian, the decision metrics from the previous time instant and the current decision metric are added and stored as,

$$
\Lambda^{\prime}\left(\tilde{u}_{n, t}\right)=\Lambda^{\prime}\left(\tilde{u}_{n, t-1}\right)+\Lambda^{\prime}\left(\tilde{u}_{n, t} / \tilde{u}_{n, t-L}, \ldots, \tilde{u}_{n, t-1}\right)
$$

At each state, only one survivor path corresponding to the minimum decision metric is stored, and the maximum likelihood path is found by tracing back the path corresponding to the surviving states. The trace back occurs after all $T_{c}$ symbols have been processed. The detected symbols correspond to the outputs of the transitions in the maximum likelihood path. The channel cancellation is inherent in the trellis; explicit estimates of the channel may be easily compted using (6) after data detection is complete.

\section{Simulation Results}

The performance of the proposed channel estimation method was simulated for an OFDM system based on IEEE 802.11a wireless local area network (WLAN) standard specification [14]. The standard specifies 48 data sub-carriers, placed over a $20 \mathrm{MHz}$ channel using a 64 point IFFT. The sub-carriers reserved for the pilots were left unused. The transmission was packetized with the channel remaining fixed over one packet. The number of OFDM symbols in the packet varied with the coherence time $T_{c}$. The channel is block fading over the $T_{c}$ symbols and its filter tap coefficients were generated according to a power-delay profile described by (2). A minimum coherence time of $12 \mu \mathrm{s}$ at $5.2 \mathrm{GHz}$ was considered.

\section{A. Optimization of pilot power fraction $\rho$}

The average pilot power fraction $\rho$ is the critical parameter that determines the performance of the system and needs to be optimized. In the first set of simulations, we fix the averaging order, $L=4$ and vary the pilot power for a given signal to noise ratio (SNR). It can be observed from Fig. 4, the BER 


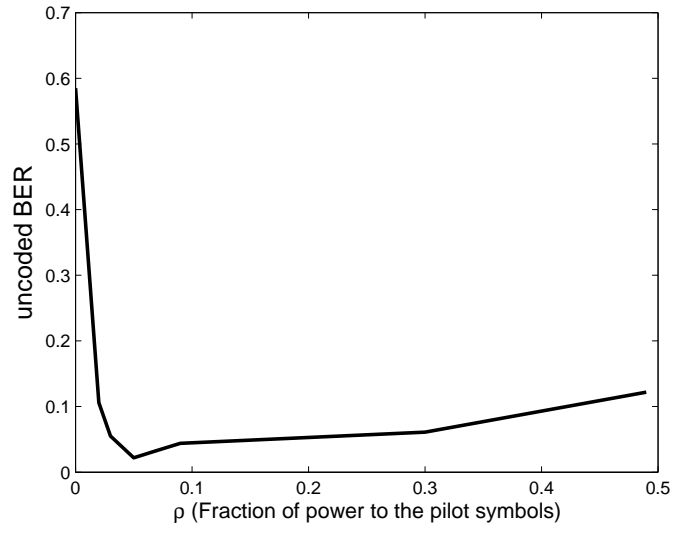

Fig. 4. Optimization of the power $\rho$ of the superimposed pilot.

is minimized for $\rho=0.05$. It should also be noted that the numerical optimization, does not minimize the BER at $\rho=$ 0 , which means that a small amount of pilot energy superimposed on the data actually aids in the coherent detection of the data.The initial reduction in the BER with small increase in $\rho$ is concurrent with the fact that the quality of the channel estimate (mean squared error) improves with increasing pilot power. However, for $\rho>0.5$, the increase in BER is due to the decrease in the effective SNR of the data symbols. The optimal $\rho$ concurs with the result in [8] which states that the BER is minimized when the pilot power is approximately $5 \%$ of the total transmit power. Therefore, we super-imposed the pilots at $5 \%$ of the total transmit power for all our simulations.

\section{B. BER Performance}

We now demonstrate the effectiveness of channel tracking using the proposed algorithm against that of the training based channel estimation scheme for an uncoded OFDM system with QPSK modulation. The pilot power was set at the optimal value of $5 \%$ of the total transmit power. Training based channel estimation in 802.11a use the transmitted long and short training symbols in the preamble of the packet to estimate the channel. Since the channel is block fading, the channel estimate is used to correct for the channel in the $T_{c}$ OFDM symbols that follow. It can be clearly seen in Fig. 5 that using super-imposed pilots gives lower bit error rates with increasing averaging order $L$ as opposed to the conventional tracking scheme employed in the 802.11a networks. We also plot the BER curve for a genie based receiver. Since the genie based receiver has perfect knowledge of the channel, it lower bounds the performance of any receiver. The BER curve for a genie receiver using QPSK modulation can be analytically characterized as,

$$
P_{Q P S K}(E)=\frac{1}{2}\left(1-\sqrt{\frac{\bar{\gamma}_{b}}{\bar{\gamma}_{b}+2}}\right)
$$

where, $\bar{\gamma}_{b}=\frac{(1-\rho)}{N_{0}} \sigma_{f}^{2}$, is the average signal to noise ratio. A sketch of the derivation is given in the Appendix and a more detailed version can be found in [15].

Clearly, with super-imposed training, we bridge the performance gap between training and genie based receivers. For

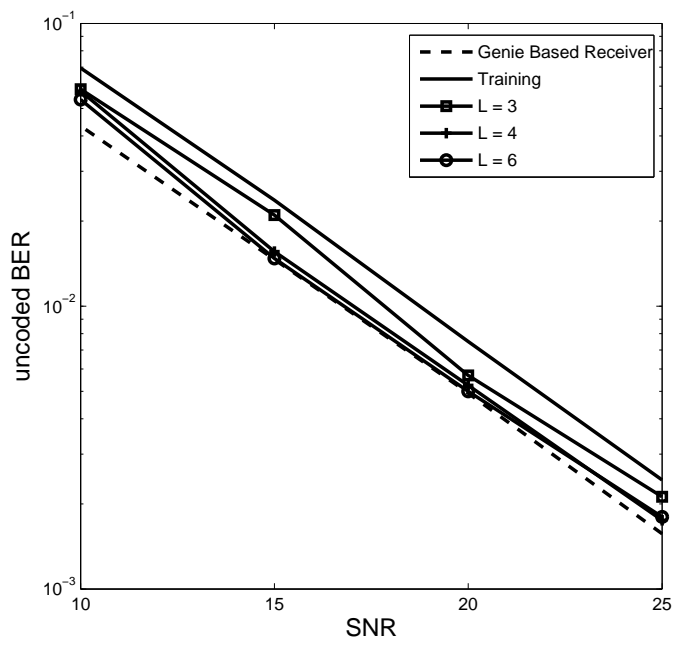

Fig. 5. Comparison of the performance of the proposed receiver with the training based method used in 802.11a and the BER bound in (10).

averaging order, $L=6$, the performance of the proposed receiver nearly equals the genie based receiver performance. Thus, increasing the averaging order beyond 6 results in only a marginal improvement in performance. Since the complexity of the Viterbi algorithm is of the order $O\left(M^{L}\right)$, operating at averaging order $L=6$ might be expensive. However, we see that reducing the averaging order to 4 , results in a very small loss in performance, but substantial savings in terms of complexity. Hence, the proposed receiver offers graceful trade-off of complexity with performance.

The BER of the proposed receiver with channel coding is plotted in Fig. 6 for averaging orders, $L=2,3,4,6$. A rate $\frac{1}{2}$ convolution code with generator matrix $\left[\begin{array}{cc}133_{8} & 171_{8}\end{array}\right]$ was used. As in the uncoded case, the performance of the receiver with channel coding at averaging order $L=6$, almost equals that of the genie based receiver.

It was also seen that the performance curves plotted did not change with changing RMS delay spread or the coherence time of the channel. The RMS delay spread is adequately compensated by the length of the cyclic prefix in the OFDM symbol, ensuring no ISI. Therefore no-ISI ensures the invariance of the performance to the RMS delay spread. The invariance in performance to the coherence time is due to the fact that changes in coherence time changes the number of OFDM symbols in the packet over which the channel is constant, and the MLSE is independent of the packet length. The performance plots for both the results will be given in the final paper.

\section{CONCLUSions}

We proposed a channel estimation algorithm using superimposed pilots for OFDM systems. The primary advantages of the proposed methods are:

- No bandwidth expansion, since the pilots are superimposed on the data.

- Transmitting super-imposed pilots provide robust channel estimation for fast-varying channels. 


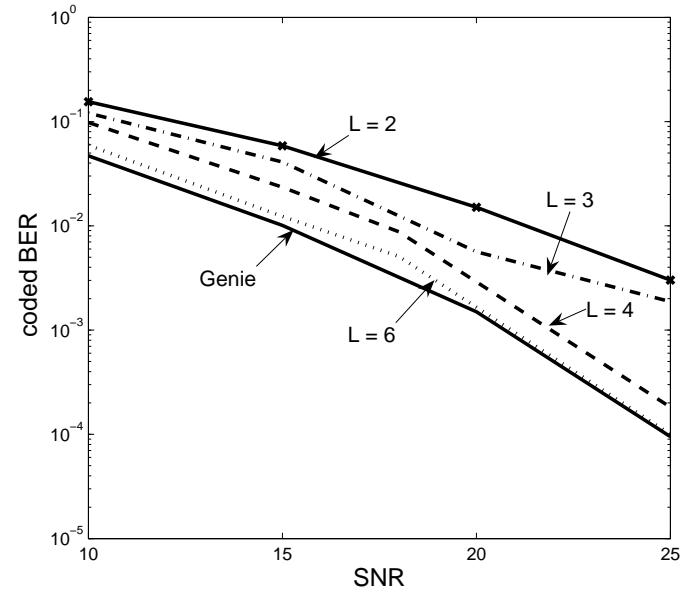

Fig. 6. Performance of the proposed receiver with rate $\frac{1}{2}$ channel coding

- The estimation algorithm is invariant to the channel's coherence time and the number of multi-paths.

Also, super-imposed PN-sequences have been used for time, phase, frequency and frame synchronization in OFDM systems [16]. The proposed channel estimation algorithm could also be applied to UWB systems where the number of multi-paths are irresolvable. Future work should focus on optimizing pilot sequences and placement, exploiting the channel's frequency response correlation across sub-carriers and extension to systems with spatial diversity.

\section{REFERENCES}

[1] A. Bahai and B. Saltzberg, Multicarrier Digital Communications: Theory and Applications of OFDM. New York: Kluwer Academic, 1999.

[2] S. Coleri, M. Ergen, A. Puri, and A. Bahai, "Channel estimation techniques based on pilot arrangement in OFDM systems," in IEEE Transactions on Broadcasting, vol. 48, no.3, September 2002, pp. 223229.

[3] H. Liu, G. Xu, L. Tong, and T. Kailath, "Recent developments in blind channel equalization : From cyclostationarity to subspaces," in Signal Processing, vol. 50, no.1-2, April 1996, pp. 83-99.

[4] B. Muquet, M. de Courville, and P. Duhamel, "Subspace based blind and semi-blind channel estimation for OFDM systems," in IEEE Transactions on Signal Processing, vol. 50, no.7, July 2002, pp. 1699-1712.

[5] A. Petropulu, R. Zhang, and R. Lin, "Blind OFDM channel estimation through simple linear precoding," in IEEE Transactions on Wireless Communications, vol. 3, no.2, March 2004, pp. 647-655.

[6] C. Kastenholtz and W. Birkemeier, "A simultaneous information transfer and channel sounding modulation technique for wide-band channels," in IEEE Transactions on Communication Technology, June 1965, pp. 162175.

[7] B. Farhang-Boroujeney, "Pilot-based channel identification: proposal for semi-blind identification of communication channels," in IEE Electronic Letters, vol. 31, no.13, June 1995, pp. 1044-1046.

[8] P. Hoeher and P. Tufvesson, "Channel estimation with superimposed pilot sequence," in Proceedings of IEEE Global Communications Conference '99, December 1999, pp. 2162-2166.

[9] G. Zhou, M. Viberg, and T. Mckelvey, "Super-imposed periodic pilots for blind channel estimation," in Proceedings of 35th Asilomar Conference on Singals, Systems, and Computers, Pacific Grove, CA, November 2001, pp. 653-657.

[10] N. Chen and G. Zhou, "A superimposed periodic pilot scheme for semiblind channel estimation of OFDM techniques," in Proccedings of the 10th IEEE DSP Workshop, Pine Mountain, GA, October 2002, pp. 362 365.
[11] J. Tugnait and W. Luo, "On channel estimation using superimposed training and first order statistics," in Proceedings of IEEE International Conference on Acoustic, Speech, Signal Processing, Hong Kong, China, April 2003, pp. 624-627.

[12] J. Terry and J. Heiskala, OFDM Wireless LANs: A theoretical and Practical Guide, 1st ed. SAMS PUBLISHING, 2001.

[13] J. Proakis, Digital Communications, 4th ed. McGraw Hill, 2001.

[14] IEEE, "Wireless LAN medium access control (MAC) and physical layer (PHY) specifications," in IEEE Standard 802.11a, no. Part 11, 1999.

[15] K. Josiam, Channel Estimation using super-imposed pilots in OFDM systems. M.S.Thesis, Southern Methodist University, December, 2004.

[16] F. Tufvesson, M. Faulkner, P. Hoeher, and O. Edfors, "OFDM time and frequency synchronization by spread spectrum pilot technique," in Proceedings of the Eigth Communication Theory Mini-Conference in conjunction with IEEE ICC '99, Vancouver, Canada, June 1999, pp. 115-119.

[17] X. Tang, M.-S. Alouini, and A. Goldsmith, "Effect of channel estimation error on M-QAM BER performance in Rayleigh fading," in IEEE Transactions on Communications, vol. 47 no. 12, December 1999, pp. $1856-1864$.

\section{APPENDIX}

Let $\hat{f}_{n}$ be the estimate of the channel computed for any given subcarrier. For a given $\hat{f}_{n}$, the transmitted symbol can be recovered from (1) as,

$$
\hat{u}_{n, t}=\frac{f_{n}}{\hat{f}_{n}}\left(u_{n, t}+p_{n, t}\right)+\frac{z_{n, t}}{\hat{f}_{n}}-p_{n, t}
$$

The channel estimate $\hat{f}_{n}$ is also assumed to have a complex Gaussian distribution with zero mean and variance $\sigma_{\hat{f}}^{2}$. Thus, the amplitudes $\left|f_{n}\right|$ and $\left|\hat{f}_{n}\right|$ are Rayleigh distributed and their joint distribution is a bivariate Rayleigh distribution, $p\left(\left|f_{n}\right|,\left|\hat{f}_{n}\right|\right)$. Assume that the bit error probability conditioned on $\left|f_{n}\right|$ and $\left|\hat{f}_{n}\right|$ is $p\left(\hat{u}_{n, t} \neq u_{n, t} /\left|f_{n}\right|,\left|\hat{f}_{n}\right|\right)$. Then, we can write the average bit error rate as [17],

$$
P(E)=\int_{0}^{\infty} \int p\left(\hat{u}_{n, t} \neq u_{n, t} /\left|f_{n}\right|,\left|\hat{f}_{n}\right|\right) p\left(|f|_{n},\left|\hat{f}_{n}\right|\right) d\left|f_{n}\right| d\left|\hat{f}_{n}\right|
$$

The bits are mapped using a gray code to the real and imaginary parts of the modulated symbol. With this mapping, the demodulation is a simple sign detection of the real and imaginary parts independently to give the most and least significant bits (MSB and LSB) respectively. Due to symmetry, the BERs of both the MSB and LSB are equal. Hence, the average BER conditioned on $\left|f_{n}\right|$ and $\left|\hat{f}_{n}\right|$ is,

$$
\begin{aligned}
p\left(\hat{u}_{n, t} \neq u_{n, t} /\left|f_{n}\right|,\left|\hat{f}_{n}\right|\right) & =\frac{1}{2}\left[p_{M S B}(E)+p_{L S B}(E)\right] \\
& =p_{M S B}(E)
\end{aligned}
$$

Since the noise variance is $\frac{\sigma_{z}^{2}}{\left|\hat{f}_{n}\right|^{2}}$, we can write the conditional bit error probability of the MSB conditioned on knowing $\left|f_{n}\right|$ and $\left|\hat{f}_{n}\right|$ as [13],

$$
p_{M S B}(E)=Q\left(\frac{\frac{\left|f_{n}\right|}{\left|\hat{f}_{n}\right|}}{\frac{\sigma_{z}}{\left|\hat{f}_{n}\right|}}\right)=Q\left(\frac{\left|f_{n}\right| d}{\sigma_{z}}\right)
$$

The average energy in the constellation is $2 d^{2}$ and can be expressed as $d=\sqrt{\frac{(1-\rho) E_{b}}{2}}$. Substituting for $d^{2}$ and $\sigma_{z}=$ 
$\sqrt{\frac{N_{0}}{2}}$ in (14), the conditional bit error rate when the MSB is in error is given by,

$$
p_{M S B}(E)=Q\left(\sqrt{\frac{\left|f_{n}\right|^{2}(1-\rho) E_{b}}{N_{0}}}\right)
$$

Since the conditional distribution is independent of $\left|\hat{f}_{n}\right|$, the average bit error rate for QPSK modulated symbols is obtained by averaging the conditional BER over the distribution of $\left|f_{n}\right|$.

$$
P_{Q P S K}(E)=\int_{0}^{\infty} Q\left(\sqrt{\frac{\left|f_{n}\right|^{2}(1-\rho) E_{b}}{N_{0}}}\right) p\left(\left|f_{n}\right|\right) d\left|f_{n}\right|
$$

where, $p\left(\left|f_{n}\right|\right)$ is the Rayleigh distribution given by $p\left(\left|f_{n}\right|\right)=$ $\frac{2\left|f_{n}\right|}{\bar{\gamma}_{b}} e^{\left(-\frac{\left|f_{n}\right|^{2}}{\bar{\gamma}_{b}}\right)}$ where, $\bar{\gamma}_{b}=\frac{(1-\rho) E_{b}}{N_{0}} \sigma_{f}^{2}$, is the average signal to noise ratio. The integral in (16) evaluates to the following expression [13],

$$
P_{Q P S K}(E)=\frac{1}{2}\left(1-\sqrt{\frac{\bar{\gamma}_{b}}{\bar{\gamma}_{b}+2}}\right) .
$$

